\title{
Population structure and diversity of the pathogenic fungus Aspergillus fumigatus isolated from different sources and geographic origins
}

\author{
Esperanza Duarte-Escalante', Gerardo Zúñiga², Oscar Nava Ramírez¹, Susana Córdoba3 \\ Nicolás Refojo ${ }^{3}$, Roberto Arenas ${ }^{4}$, Laurence Delhaes ${ }^{5}$, María del Rocío Reyes-Montes ${ }^{1 /+}$
}

\begin{abstract}
${ }^{1}$ Laboratorio de Micología Molecular, Departamento de Microbiología-Parasitología, Facultad de Medicina, Universidad Nacional Autónoma de México, 04510 México, DF, México ²Departamento de Zoología, Escuela Nacional de Ciencias Biológicas, Instituto Politécnico Nacional, México, DF, México ${ }^{3}$ Departamento Micología INEI ANLIS Dr. Carlos G. Malbrán, Buenos Aires, Argentina ${ }^{4}$ Departamento de Dermatología, Hospital General Dr. Manuel Gea González, General Hospital, México, DF, México ${ }^{5}$ EA3609, Lille 2 University, Pasteur Institute of Lille, Lille, France
\end{abstract}

Fifty-five clinical and environmental Aspergillus fumigatus isolates from Mexico, Argentina, France and Peru were analyzed to determine their genetic variability, reproductive system and level of differentiation using amplified fragment length polymorphism markers. The level of genetic variability was assessed by measuring the percentage of polymorphic loci, number of effective alleles, expected heterozygocity and by performing an association index test $\left(\mathrm{I}_{\alpha}\right)$. The degree of genetic differentiation and variation was determined using analysis of molecular variance at three levels. Using the paired genetic distances, a dendrogram was built to detect the genetic relationship among alleles. Finally, a network of haplotypes was constructed to determine the geographic relationship among them. The results indicate that the clinical isolates have greater genetic variability than the environmental isolates. The $I_{A}$ of the clinical and environmental isolates suggests a recombining population structure. The genetic differentiation among isolates and the dendrogram suggest that the groups of isolates are different. The network of haplotypes demonstrates that the majority of the isolates are grouped according to geographic origin.

Key words: Aspergillus fumigatus - AFLP - genetic diversity - polymorphisms

Aspergillus fumigatus Fresenius is the causal agent of aspergillosis, a widely distributed fungal disease. It lives in soil, where it grows among organic remains. This fungus contributes to the decomposition of organic matter, such as compost (humus) and fodder, while also playing an active role in the recycling of carbon and nitrogen sources (Latgé 1999, 2003).

A. fumigatus produces large amounts of spores on its conidial head. The dispersion mechanism of these spores is very simple, as the conidia are released into the environment through air currents. The conidia range between 2-3 $\mu \mathrm{m}$ in diameter (Latgé 1999, 2003). On average, humans inhale hundreds of these infectious propagules daily, yet inhalation of the conidia by immunocompetent hosts rarely shows adverse effects since their immune systems are able to eliminate the fungus. Disease occurs, however, when the host response is either too strong or too weak. In immunocompromised hosts, A. fumigatus represents a major cause of morbidity and mortality. In the last decade, this patient population is expanding, even in developed countries, due to the increased use of transplantation, to the develop-

Financial support: DGAPA (DGAPA-UNAM-IN224706-3)

+ Corresponding author: remoa@servidor.unam.mx

Received 30 July 2008

Accepted 10 March 2009 ment of immunosuppressive and myeloablative therapies for autoimmune and neoplastic disease, and to the human immunodeficiency virus/AIDS pandemic (Hohl \& Feldmesser 2007). While the clinical epidemiology, human risk factors, and treatment strategies for fungal opportunists such as Candida spp. and Aspergillus spp. are currently well codified (Eggiman et al. 2003, Singh \& Paterson 2005, Ullmann \& Cornerly 2006), relatively little is known about Aspergillus environmental circulation, reproduction or molecular epidemiology.

Molecular studies characterizing aspergillosis in others countries, especially in European countries (Fridkin \& Jarvis 1996, Menotti et al. 2005), have been increasing in recent years, but few data are available from Latin or South American countries. Several molecular methods have been evaluated for A. fumigatus strain typing and these methods include randomly amplified polymorphic DNA typing (Aufauvre-Brown et al. 1992), sequencespecific DNA primer analysis (Lin et al. 1995), polymorphic microsatellite markers (Bart-Delabesse et al. 1998, 1999, de Valk et al. 2005, 2007) and analysis of hybridization profiles with the dispersed, repetitive DNA probe Afutl restriction fragment length polymorphism (RFLP) (Neuveglise et al. 1996, Chazalet et al. 1998). Those molecular typing techniques allow elucidation of the epidemiology of Aspegillus infections and investigation of potential case clusters, but they can also provide information to analyze whether the micromycete reproduction mode affects antifungal susceptibility evolution, especially in terms of resistance gene dissemination.

Although numerous papers were published recently on the application of several typing techniques to A. fumigatus 
isolates, only some authors attempted to examine the reproductive mode of A. fumigatus populations and the conclusions drawn by them were controversial (Girardin et al. 1994, Fridkin \& Jarvis 1996, Lasker 2002, Levdansky et al. 2007). In Latin America, there is very little information on these different aspects of the epidemiology of aspergillosis. The most outstanding epidemiological data are summarized and reported in retrospective studies on patients diagnosed with fungal infections or correspond to case reports. The observation of high levels of genetic diversity within this supposedly asexual species (Varga \& Tóth 2003).

To address these concerns, we performed a molecular analysis of A. fumigatus isolates to examine genetic variation among clinical and environmental A. fumigatus isolates from different geographical origins [Mexico (MX), Argentina (AR), France (FR) and Peru (PE)] to determine mode of fungal reproduction and the contribution of each variable to the disease epidemiology, in order to improve our control and prevention therapy guide lines.

We choose the AFLP (amplified fragment length polymorphisms) technique, because it represents a highly discriminatory method at the intraspecific level and previously has been shown to have good discriminatory power for fungal strain differentiation (O'Donnell et al.
2004, Delhaes et al. 2008). In AFLP analyses, fragments are amplified from random locations throughout an organism's genome in a highly reproducible manner (Vos et al. 1995, O’Donnell et al. 2004, Delhaes et al. 2008).

\section{MATERIALS AND METHODS}

Isolates - Fifty five isolates of A. fumigatus were used (Table I). A. fumigatus isolates were identified by their macroscopic or microscopic appearance and their ability to grow at $48^{\circ} \mathrm{C}$. The fungal strains were cultured on YPD at $37^{\circ} \mathrm{C}$ for $18 \mathrm{~h}$ and DNA extraction was performed as described by Reyes-Montes et al. (1999).

AFLP - AFLP analysis was performed as described by Vos et al. (1995). Briefly, DNA was restricted with the endonucleases EcoRI and MseI. After restriction, adaptors were ligated to the resulting fragments. The resulting fragments were preamplified with primers E (5'-GACTGCGTACCAATTC- $\left.3^{\prime}\right)$ and M (5'-GACGATGAGTCCTGAGTAA-3'), followed by a second, selective PCR. The selective primers were identical to primer E or M but were extended with specific two or three nucleotides at the 3 '- terminus. Eight primer combinations were used: $\mathrm{E}+\mathrm{AA}: \mathrm{M}+\mathrm{CTC} ; \mathrm{E}+\mathrm{AC}: \mathrm{M}+\mathrm{CAC}$; $\mathrm{E}+\mathrm{AA}: \mathrm{M}+\mathrm{CAT} ; \quad \mathrm{E}+\mathrm{AC}: \mathrm{M}+\mathrm{CTC} ; \mathrm{E}+\mathrm{AA}: \mathrm{M}+\mathrm{CTG}$;

TABLE I

Source and geographic origin of Aspergillus fumigatus isolates

\begin{tabular}{|c|c|c|c|c|c|}
\hline Isolate & Source & Geographic origin & Isolate & Source & Geographic origin \\
\hline MM-7 & Environment & MX & $951740 \mathrm{a}$ & Clinical & $\mathrm{AR}$ \\
\hline MM-8 & Environment & MX & 951741 & Clinical & $\mathrm{AR}$ \\
\hline MM-9 & Clinical & MX & 951744 & Clinical & AR \\
\hline MM-10 & Clinical & MX & 951745 & Clinical & AR \\
\hline MM-11 & Clinical & MX & 951746 & Clinical & $\mathrm{AR}$ \\
\hline MM-32 & Clinical & MX & 951747 & Clinical & AR \\
\hline MM-33 & Clinical & MX & 951748 & Clinical & $\mathrm{AR}$ \\
\hline MM-34 & Clinical & MX & 88248 & Clinical & AR \\
\hline MM-35 & Clinical & MX & 90370 & Clinical & $\mathrm{AR}$ \\
\hline MM-36 & Clinical & MX & 982928 & Clinical & AR \\
\hline MM-37 & Clinical & MX & 993315 & Clinical & $\mathrm{AR}$ \\
\hline MM-38 & Clinical & MX & 9272 & Clinical & AR \\
\hline MM-39 & Clinical & MX & 8571 & Clinical & AR \\
\hline MM-45 & Clinical & MX & 6578 & Clinical & AR \\
\hline MM-46 & Clinical & MX & 51435 & Clinical & $\mathrm{PE}$ \\
\hline Amb III & Environment & $\mathrm{AR}$ & 51594 & Clinical & PE \\
\hline Amb V & Environment & AR & 53027 & Clinical & $\mathrm{PE}$ \\
\hline Amb VIII & Environment & AR & 53097 & Clinical & PE \\
\hline 951740 & Clinical & AR & Af- 8 & Clinical & FR \\
\hline 951744 & Clinical & AR & Af-11 & Clinical & FR \\
\hline 951746 & Clinical & AR & Af-15 & Clinical & FR \\
\hline 951722 & Clinical & $\mathrm{AR}$ & Af- 22 & Clinical & FR \\
\hline 951733 & Clinical & AR & Af- 26 & Clinical & FR \\
\hline 951734 & Clinical & $\mathrm{AR}$ & Af-29 & Clinical & FR \\
\hline 951736 & Clinical & AR & Af-34 & Clinical & FR \\
\hline 951737 & Clinical & $\mathrm{AR}$ & Af-35 & Clinical & FR \\
\hline 951738 & Clinical & $\mathrm{AR}$ & Af-41 & Clinical & FR \\
\hline 951739 & Clinical & AR & & & \\
\hline
\end{tabular}

AR: Argentina; FR: France; MX: Mexico; PE: Peru. 
E+AC:M+CAT; E+AA:M+CAC; E+AC:M+CTG. Primer E was radioactively labelled with ATP-P ${ }^{32}$ and the amplified material was analyzed on 5\% polyacrylamide slab gels.

Statistical analysis - Reproducible bands with sizes between 100-400 bp in different samples across the gel were scored. AFLP markers were scored visually, compared with a 50 bp DNA Ladder (Invitrogen Life Technologies, Carlsbad, CA), manually coded and analyzed as binary data of presence (1) or absence (0). Genetic diversity of AFLP markers was calculated by Shannon's Index $(I)$, assuming that each phenotypic marker represents a distinct locus (Allnutt et al. 1999), and Nei's genetic diversity, using allelic frequencies (Nei 1973). Additionally, expected heterozygosity per population and average heterozygosity were calculated using allele frequencies according to Zhivotovsky's Bayesian method (Zhivotovsky 1999). Analysis of molecular variance (AMOVA) using FAMD v $1.1 \beta$ (Schlüter \& Harris 2006) was performed to partition the molecular variance into different hierarchical levels. AMOVA analysis was carried out to test differences among isolations, among isolations within countries and between isolation sources (clinical and environmental).

The statistical significance of the molecular variance partitioning and the associated estimates of $\varphi$-statistics were assessed by conducting 10,000 random permutations of the data (Excoffier et al. 1992). Pairwise genetic distances among groups of isolates were calculated using Nei's method (Nei 1973). The groups were formed according to their geographic origin (countries) and isolation source (clinical and environmental). The genetic relationship among them was established by unweighted paired group method using the arithmetic mean (UPGMA - PopGene 1.31 program) (Yeh et al. 1999). The distortion of the inferred tree was estimated by means of the cophenetic correlation coefficient (CCCr) using Mantel's test (Manly 1997). In addition, a haplotype network was built to evaluate the genealogical relationships among AFLP haplotypes by the Median-Joining method (Bandelt et al. 1999) implemented in NETWORK 4.2.0.1 (htt://www.fluxus-engineering.com, Polzin \& Daneschmand 2003). The parameters used were epsilon $=0,1 / 1$ transitions-transvertions weight, 10 characters weight, and connexion criteria. To distinguish between clonal and recombinant structures, we used the index of association $\left(I_{\mathrm{A}}\right)$ (Maynard-Smith et al. 1993), a statistical test that measures the degree of non-random association between alleles at different loci (linkage disequilibrium), implemented in LIAN v 3.5 (Haubold \& Hudson 2000).

\section{RESULTS}

Using the eight combinations of primers, a total of 105 AFLP markers were amplified. Genetic variation levels of isolates are shown according to geographic and isolation source in Table II. In general, the estimates of genetic variability indicate that the clinical isolates are more variable than the environmental ones. Average polymorphism of isolates varied from $81.90 \%$ (AR) to $22.86 \%$ (PE) and from $18.10 \%(\mathrm{AR})$ to $6.67 \%$ (MX), respectively, for clinical and environmental isolates. The number of effective alleles, as well as values for genetic diversity estimated from the $I$ - an estimator not sensible to the number of isolates analyzed - and the average heterozygosity showed the same tendency for both clinical and environmental isolates. The AMOVA shows the structure of the genetic variation of the group of isolates analyzed. The percentage of variation shared among the clinical and environmental isolates was very low $(9.05 \%)$ and the genetic differentiation was statistically significant $(F s t=$ $0.253, \mathrm{p}<0.001)$. Between these two groups, the shared variation among geographically grouped isolates was slightly greater $(16.22 \%)$, but the differentiation was also statistically significant $(F s t=0.178, \mathrm{p}<0.001)$. Finally, the shared variation among the isolates from the same country was high (74.73\%) and their genetic differentiation did not differ from zero ( $F s t=0.091, \mathrm{p}=0.046$ ).

The $I_{A}$ value estimated from all of isolates indicates that $A$. fumigatus has a recombinant reproductive system (Table III). The analysis conducted on partial isolate groups by geographic origin and isolation source showed similar results.

The dendrogram shows the integration of two welldefined groups (Fig. 1). The first is comprised of isolates from AR and MX, which are separated into two subgroups according to the isolation source of each isolate. The second group clustered the isolates from FR and PE. The CCCr $(0.70, p<0.005)$ indicates both that the tree is a good representation of genetic relationships of the isolates and that the different groups are consistent. Lastly, the network of the total group of AFLP haplotypes shows that the number of mutations ranges between one-20 (Fig. 2). The network of haplotypes shows a large number of reticula-

\section{TABLE II}

Polymorphism, effective number of alleles, genetic diversity and expected heterozygosity of Aspergillus fumigatus isolates

\begin{tabular}{lrcrr}
\hline Population & $\mathrm{P}(\%)$ & $\mathrm{n}_{\mathrm{e}}$ & $I$ & $\mathrm{~h}$ \\
\hline $\mathrm{MX}^{a}$ & 6.67 & $1.0471 \pm 0.1253$ & $0.0403 \pm 0.1071$ & $0.0276 \pm 0.0733$ \\
$\mathrm{MX}^{b}$ & 72.38 & $1.5197 \pm 0.1083$ & $0.4243 \pm 0.0785$ & $0.2912 \pm 0.0559$ \\
$\mathrm{AR}^{a}$ & 18.10 & $1.1225 \pm 0.1644$ & $0.1038 \pm 0.1305$ & $0.0704 \pm 0.0898$ \\
$\mathrm{AR}^{b}$ & 81.90 & $1.6128 \pm 0.0761$ & $0.4890 \pm 0.0524$ & $0.3379 \pm 0.0379$ \\
$\mathrm{FR}^{b}$ & 70.48 & $1.4726 \pm 0.1292$ & $0.3945 \pm 0.0951$ & $0.2683 \pm 0.0678$ \\
$\mathrm{PE}^{b}$ & 22.86 & $1.1657 \pm 0.1624$ & $0.1361 \pm 0.1278$ & $0.0935 \pm 0.0888$ \\
\hline
\end{tabular}

$a$ : environmental isolates; $b$ : clinical isolates; AR: Argentina; FR: France; h: heterozygosity; I: genetic diversity, Shannon's index; MX: Mexico; $n_{\mathrm{e}}$ : effective number of alleles; P: polymorphism; PE: Peru. 
TABLE III

Reproductive structure of Aspergillus fumigatus isolates

\begin{tabular}{llc}
\hline Groups & Isolates & $I_{A}$ \\
\hline MX, AR & Enrironmental & $0.0111(<0.05)$ \\
MX, AR, & & \\
FR, PE & Clinical & $0.0378(<0.05)$ \\
MX & Clinical and environmental & $0.0650(<0.05)$ \\
AR & Clinical and environmental & $0.0469(<0.05)$ \\
FR & Clinical & $0.0586(<0.05)$ \\
PE & Clinical & $0.0087(<0.05)$
\end{tabular}

AR: Argentina; FR: France; $I_{A}$ : index of association; MX: Mexico; PE: Peru.

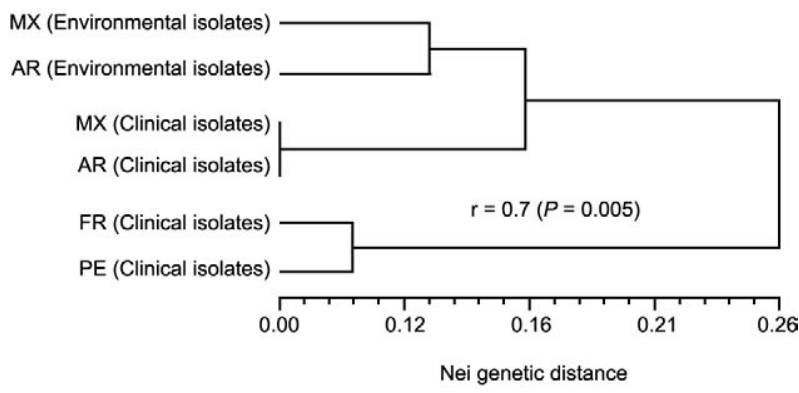

Fig. 1: the dendrogram generated between the environmental and clinical isolates from the different countries constructed using UPGMA pairwise genetic distance (Nei 1973). AR: Argentina; FR: France; MX: Mexico; PE: Peru.

tions among the isolates studied, exhibiting a clear association among the majority of isolates from MX (group A), AR (group B) and PE (group C) to their geographic origin, with some exceptions. The isolates from FR and some from AR are highly variable, which determines their position to be more irregular. However, all the isolates from PE are in the same group.

\section{DISCUSSION}

In this study, the AFLP technique used with eight selective base combinations, was found to be useful for distinguishing among A. fumigatus strains obtained from the environment and patients, as well as for identifying the structures of populations and their relationships with their geographical origin, according to previous published results (Warris et al. 2003, Bonin et al. 2007, de Valk et al. 2007).

The results of this study demonstrate that the levels of genetic variation between the A. fumigatus clinical isolates had the highest values, with the exception of those from Peru. In contrast, the environmental isolates had the lowest genetic variation values. When we analyzed the clinical and environmental isolates of $A$. fumigatus from different geographic origins, the genetic differentiation values found for the clinical isolates were greater than those found for the environmental ones, showing that they are genetically different groups. The dendro-

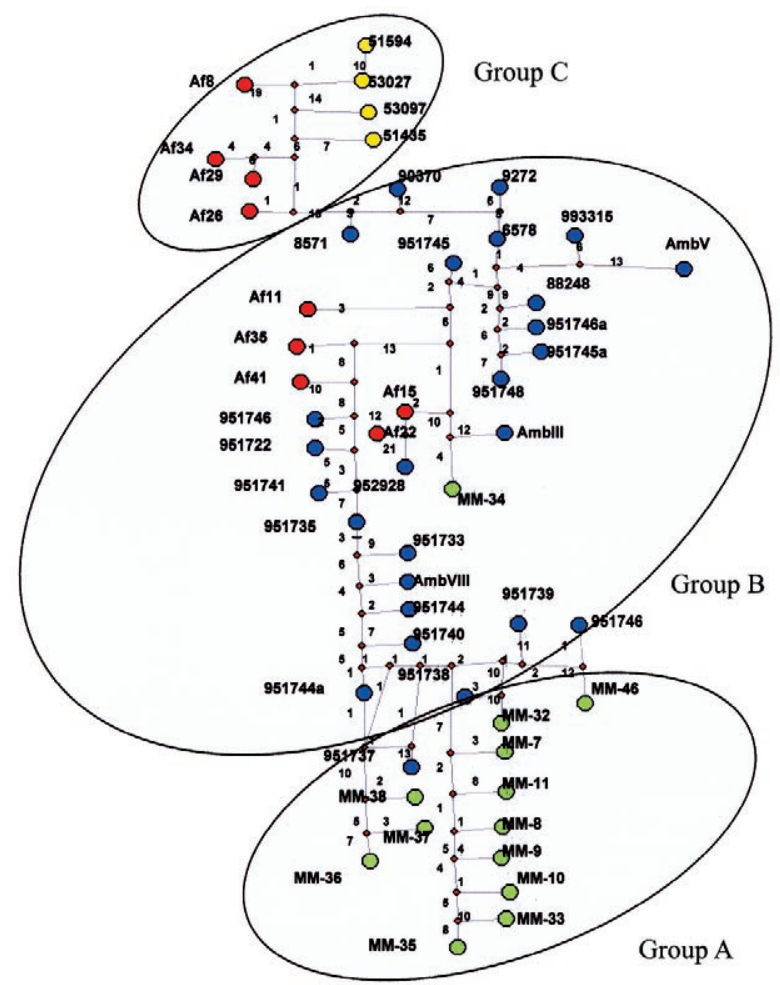

Fig. 2: median-joining network analysis of Aspergillus fumigatus isolates. The green circles correspond to the isolates from Mexico; blue circles correspond to the isolates from Argentina; red circles correspond to the isolates from France; yellow circles correspond to the isolates from Peru.

gram, based on the Nei genetic distance, groups the $\mathrm{MX}$ and AR isolates in a single group, but subclusters the environmental isolates separately from the clinical isolates, indicating that the environmental isolates from MX and AR are genetically different from the clinical ones. On the other hand, the clinical isolates from FR and PE constitute another group, showing that they do not share many genetic characteristics with the MX and AR isolates. Despite the low number of environmental isolates $(n=5)$ as compared with the large number of clinical isolates $(\mathrm{n}=50)$, genetic differences observed in our study may be explained by (i) a colonization prior to a patient's hospitalization that develops into an infection after the patient becomes immunosuppressed or (ii) concomitant infection by multiple strains where only one survived or has been isolated and typed. Alternatively, genetic differences could be explained by studies reporting that patients inhaling hundreds of Aspergillus genotypes from the environment have developed polyclonal invasive aspergillosis more frequently than expected. According to these studies, the percentage of polyclonal infections could be established in $50-75 \%$ of the cases; in certain patients, up to five different genotypes could be detected (Bart-Delabesse et al. 1999, Bertout et al. 2001). This also confirms that individuals could be exposed to a great variety of Aspergillus genotypes in the environment (Chazalet et al. 1998, Menotti et al. 2005). 
These findings suggest that only isolates that adapt to the host will grow. In addition, genetic differences can result from the ability of the isolates that enter the patient to reactivate their virulence, which changes their genotype and generates more variability in relation to the original strain (Latgé 2003). Another explanation is that environmental isolates recovered in the laboratory are not necessarily those that infect patients.

The AMOVA analysis suggests that the genetic variation of Aspergillus isolates is highly structured by geographic origin and isolation source. This structure could be by natural selection, as suggested by the observed recombinant reproductive structure and revealed by the $I_{A}$ estimated in each isolate group, where all $I_{A}$ values were found to be approximately zero (Table III). It was previously thought that the variability seen in A. fumigatus could be attributed to the possibility of parasexual genetic recombinations (Pál et al. 2007). However, these are infrequent and are limited to isolates within the same vegetative compatibility group. Another possibility, which most authors prefer, is the existence of unknown sexual forms or variability produced by previous sexual recombinations at the time when A. fumigatus was selected as a species (Varga 2003). These explanations, supported by meta-analysis carried out with conglomerate analysis and association indexes, show that the strains that are geographically related are more genetically similar than those that are not. These findings reaffirm the hypothesis that variability is due to an ancient recombination and some selective pressure (Varga 2003). These hypotheses are reinforced by the work of Paoletti et al. (2005), which shows that A. fumigatus isolates containing the complementary alpha-domain MAT gene were identified, and showed the expression of MAT1-1 and MAT1-2 and of genes encoding for sex pheromones and pheromone receptors. These results indicate that $A$. fumigatus has a recent evolutionary history of sexual recombination and might have the potential for sexual reproduction. This form of reproduction has now been confirmed with the finding that a sexual reproductive cycle in this fungus causes the production of cleistothecia and ascospores. When found in its teleomorphic (sexual) state, it was included in the Neosartorya genus on the basis of the phylogenetic and morphologic relation of its sexual forms (Rydholm et al. 2006, Samson et al. 2007) and named Neosartorya fumigata $\mathrm{O}^{\prime}$ Gorman, Fuller \& Dyer sp. nov. (O'Gorman et al. 2009). The recent confirmation of a sexual cycle in A. fumigatus explains the great genotypic diversity found among the isolates studied.

When a review of the relationship between isolate haplotypes was performed, it was found that the majority of the isolates were grouped according to their geographic origin, and could be defined by three large groups. Group $\mathrm{C}$ contained the majority of the isolates from FR and all of those from PE, which seem to cluster into their own sub-group. On the other hand, group B showed that the AR isolates cluster with some isolates from FR and MX, leading to a relationship close to AR isolates' paraphyletic behaviour. Many reticulations with one-20 mutational changes are seen in the network of isolates, reflecting again the large variability among them, similar to published data by Warris et al. (2003). The current study showed that both clinical and environmental isolates of A. fumigatus display genetic diversity, reinforcing conclusions reached by other investigators (Warris et al. 2003). These facts could have repercussions for the diagnosis and treatment of patients with aspergillosis, since it has become more common to find great variation within this species. Changes due to mutations or recombinations can occur and do reflect the Aspergillus resistance to anti-mycotic treatments (DíazGuerra et al. 2000, Trama et al. 2005).

As Aspergillus infections remain difficult to treat (Denning et al. 1997), and the outcomes, especially in immunocompromised patients, are very poor, prevention is ever more important. Therefore, understanding the epidemiology, evolution and ability of this fungus to adapt should help in its future management. Moreover, it is pertinent to point out that in order to obtain greater knowledge about genetic and structural diversity of $A$. fumigatus populations it is important to obtain a greater number of environmental samples. Further molecular studies analyzing clinical and environmental Aspergillus isolates will be useful in determining the genetic relationships between isolates responsible for infections occurring in the same institution, in revealing whether this filamentous fungus is able to develop different routes of transmission (air and/or water) as has been proposed (Warris et al. 2003) and which reproduction mode is primarily used by Aspergillus.

\section{ACKNOWLEDGEMENTS}

To the Dra. B. Bustamante, for their generous contribution of A. fumigatus isolates from Peru, used in this study.

\section{REFERENCES}

Allnut TR, Newton AC, Lara A, Premoli A, Armesto JJ, Vergara R, Gardner M 1999. Genetic variation in Fitzroya cupressoides (alerce), a threatened South American conifer. Mol Ecol 8: 975-987.

Aufauvre-Brown A, Cohen J, Holden DW 1992. Use of randomly amplified polymorphic DNA markers to distinguish isolates of Aspergillus fumigatus. J Clin Microbiol 30: 2991-2993.

Bandelt H, Forster P, Röhl AA 1999. Median-Joining networks for inferring intraspecific phylogenies. Mol Biol Evol 16: 37-48.

Bart-Delabesse E, Cordonnier C, Bretagne S 1999. Usefulness of genotyping with microsatellite markers to investigate hospitalacquired invasive aspergillosis. J Hosp Infect 42: 321-327.

Bart-Delabesse E, Humbert JF, Delabesse E, Bretagne S 1998. Microsatellite markers for typing Aspergillus fumigatus isolates. J Clin Microbiol 36: 2413-2418.

Bertout S, Renaud F, Barton R, Symoens F, Burnod J, Piens MA, Lebeau B, Viviani MA. Chapuis F, Bastide JM, Grillot R, Mallié M, The European Research Group on Biotype and Genotype of Aspergillus. Genetic polymorphism of Aspergillus fumigatus in clinical samples from patients with invasive aspergillosis: investigation using multiple typing methods. J Clin Microbiol 39: 1731-1737.

Bonin A, Ehrich D, Manel S 2007. Statistical analysis of amplified fragment length polymorphism data: a toolbox for molecular ecologists and evolutionists. Mol Ecol 16: 3737-3758. 
Chazalet V, Debeaupuis JP, Sarfati J, Lortholary J, Ribaud P, Shah P, Cornet M, Vu Thien H, Gluckman E, Brucker G, Latgé JP 1998. Molecular typing of environmental and patient isolates of Aspergillus fumigatus from various hospital settings. J Clin Microbiol 36: $1494-1500$

Delhaes L, Harun A, Chen SC, Nguyen Q, Slavin M, Heath CH, Maszewska K, Halliday C, Robert V, Sorrell TC, the Australian Scedosporium (AUSCEDO) Study Group, Meyer W 2008. Molecular typing of Australian Scedosporium isolates showing genetic variability and numerous S. aurantiacum. Emerg Infect Dis 14: 282-290.

Denning DW, Venkateswarin K, Oakley L, Anderson MJ, Manning NJ, Stevens DA, Warnock DA, Kelly SL 1997. Itraconazole resistance in Aspergillus fumigatus. Antimicrob Agents Chemother 41: 1364-1368.

de Valk HA, Meis JF, Curfs IM, Muehlethaler K, Mouton JW, Klaassen CH 2005. Use of a novel panel of nine short tandem repeats for exact and high-resolution fingerprinting of Aspergillus fumigatus isolates. J Clin Microbiol 43: 4112-4120.

de Valk HA, Meis JF, de Pauw BE, Donnelly PJ, Klaassen CH 2007. Comparison of two highly discriminatory molecular fingerprinting assays for analysis of multiple Aspergillus fumigatus isolates from patients with invasive aspergillosis. J Clin Microbiol 45: 1415-1419.

Díaz-Guerra TM, Mellado E, Cuenca-Estrella M, Gaztelurrutia L, Navarro JI, Tudela JL 2000. Genetic similarity among one Aspergillus flavus strain isolated from a patient who underwent heart surgery and two environmental strains obtained from the operating room. J Clin Microbiol 38: 2419-2422.

Eggiman P, Garbo J, Pittet D 2003. Epidemiology of Candida species infections in critically ill non-immunosuppresed patients. Lancet Infect Dis 3: 685-702.

Excoffier L, Smouse PE, Quattro JM 1992. Analysis of molecular variant inferred from metric distances among DNA haplotypes: application to human mitochondrial DNA restriction data. Genetics 131: 479-491.

Fridkin SK, Jarvis WR 1996. Epidemiology of nosocomial fungal infections. Clin Microbiol Rev 9: 499-511.

Girardin H, Sarfati J, Traore F, Camet DJ, Derouin F, Latgé JP 1994. Molecular epidemiology of nosocomial invasive aspergillosis. J Clin Microbiol 32: 684-690.

Haubold B, Hudson RR 2000. LIAN 3.0: detecting linkage disequilibrium in multilocus data. Bioinformatics 16: 847-848

Hohl TM, Feldmesser M 2007. Aspergillus fumigatus: principles of pathogenesis and host defense. Eukaryotic Cell 6: 1953-1963.

Lasker BA 2002. Evaluation of performance of four genotypic methods for studying the genetic epidemiology of Aspergillus fumigatus isolates. J Clin Microbiol 40: 2886-2892.

Latgé JP 1999. Aspergillus fumigatus and aspergillosis. Clin Microbiol Rev 12: 310-350.

Latgé JP 2003. Aspergillus fumigatus, a saprotrotophic pathogenic fungus. Mycologist 17: 56-61.

Levdansky E, Romano J, Shadkchan Y, Sharon H, Verstrepen KJ, Fink GR, Osherov N 2007. Coding tandem repeats generate diversity in Aspergillus fumigatus genes. Eukaryotic Cell 6: 1380-1391.

Lin D, Lehmann PF, Hamory BH, Padhye AA, Durry E, Pinner RW, Lasker BA 1995. Comparison of three typing methods for clinical and environmental isolates of Aspergillus fumigatus. $J$ Clin Microbiol 33: 1596-1601.
Manly BFJ 1997. Randomization, Bootstrap and Montecarlo methods in biology, Chapman and Hall, London, 399 pp.

Maynard-Smith J, Smith NH, O'Rourke M, Spratt BG 1993. How clonal are bacteria? Proc Natl Acad Sci USA 90: 4384-4388.

Menotti J, Waller J, Meunier O, Letscher-Bru V, Herbrecht R, Candolfi E 2005. Epidemiological study of invasive pulmonary aspergillosis in a haematology unit by molecular typing of environmental and patient isolates of Aspergillus fumigatus. J Hosp Infect 60: 61-68.

Nei M 1973. Analysis of gene diversity in subdivided populations. Proc Nat Acad Sci USA 70: 3321-3323.

Neuveglise C, Sarfati J, Latgé JP, Paris S 1996. Afut1, a retrotransposon-like element from Aspergillus fumigatus. Nucleic Acids Res 24: $1428-1434$

O'Donnell K, Sutton DA, Rinaldi MG, Magnon KC, Cox PA, Revankar SG, Sanche S, Geiser DM, Juba JH, van Burik JA, Padhye A, Anaissie EJ, Francesconi A, Walsh TJ, Robinson JS 2004. Genetic diversity of human pathogenic members of the Fusarium oxysporum complex inferred from multilocus DNA sequence data and amplified fragment length polymorphism analyses: evidence for the recent dispersion of a geographically widespread clonal lineage and nosocomial origin. J Clin Microbiol 42: 5109-5120.

O'Gorman CM, Fuller HT, Dyer PS 2009. Discovery of a sexual cycle in the opportunistic fungal pathogen Aspergillus fumigatus. $\mathrm{Na}$ ture 457: 471-474.

Pál K, van Diepeningen AD, Varga J, Hoekstra RF, Dyer PS, Debets AJM 2007. Sexual and vegetative compatibility genes in the aspergilli. Stud Mycol 59: 19-30.

Paoletti M, Rydholm C, Schwier EU, Anderson MJ, Szakacs G, Lutzoni F, Debeaupuis JP, Latgé JP, Denning DW, Dyer PS 2005. Evidence for sexuality in the opportunistic fungal pathogen Aspergillus fumigatus. Curr Biol 15: 1242-1248.

Polzin T, Daneschmand SV 2003. On Steiner trees and minimum spanning trees in hypergraphs. Oper Res Lett 31: 12-20.

Reyes-Montes MR, Bobadilla del Valle M, Martínez-Rivera MA, Rodríguez-Arellanes G, Maravilla E, Sifuentes-Osornio J, Taylor ML 1999. Relatedness analyses of Histoplasma capsulatum isolates from Mexican patients with AIDS-associated histoplasmosis by using histoplasmin electrophoretic profiles and randomly amplified polymorphic DNA patterns. $J$ Clin Microbiol 37: 1404-1408.

Rydholm C, Szakacs G, Lutzoni F 2006. Low genetic variation and no detectable population structure in Aspergillus fumigatus compared to closely related Neosartorya species. Eukaryot Cell 5: 650-657.

Samson RA, Hong S, Peterson SW, Frisvad JC, Varga J 2007. Polyphasic taxonomy of Aspergillus section Fumigati and its teleomorph Neosartorya. Stud Mycol 59: 147-203.

Schlüter PM, Harris SA 2006. Analysis of multilocus fingerprinting data sets containing missing data, Mol Eco Notes 6: 569-572.

Singh N, Paterson D 2005. Aspergillus infections in transplant recipients. Clin Microbiol Rev 18: 44-69.

Trama JP, Mordechai E, Adelson ME 2005. Detection of Aspergillus fumigatus and a mutation that confers reduced susceptibility to itraconazole and posaconazole by real-time PCR and pyrosequencing. J Clin Microbiol 43: 906-908.

Ullmann AJ, Cornerly OA 2006. Antifungal prophylaxis for invasive mycoses in high risk patients. Curr Opin Infect Dis 19: 571-576.

Varga J 2003. Mating type gene homologues in Aspergillus fumigatus. Microbiology 149: 816-819. 
Varga J, Tóth, B 2003. Genetic variability and reproductive mode of Aspergillus fumigatus. Infect Genet Evol 3: 3-17.

Vos P, Hogers R, Bleeker M, Reijans M, Van de Lee T, Hornes M, Frijters A, Pot J, Peleman J, Kuiper M, Zabeau M 1995. AFLP: a new technique for DNA fingerprinting. Nucleic Acids Res 23: 4407-4414.

Warris A, Klaassen CHW, Meis JFGM, de Ruiter MT, de Valk HA, Abrahamsen TG, Gaustad P, Verweij PE 2003. Molecular epide- miology of Aspergillus fumigatus isolates recovered from water, air, and patients shows two clusters of genetically distinct strains. J Clin Microbiol 41: 4101-4106.

Yeh FC, Boyle T, Rongcai Y, Ye Z, Xiyan JM 1999. POPGENE Version 1.31. University of Alberta. Available from: http://www.ualberta.ca/_fyeh/fyeh.

Zhivotovsky LA 1999. Estimating population structure in diploids with multilocus dominant DNA markers. Mol Ecol 8: 907-913. 